\title{
LA INFORMACIÓN FINANCIERA EN EL NEGOCIO DE LA AERONAVEGACIÓN COMERCIAL
}

\author{
FINANCIAL INFORMATION IN THE BUSINESS OF COMMERCIAL NAVIGATION
}

\author{
Ricardo J.M. Pahlen Acuña* \\ Director del Instituto de Investigaciones y Estudios Contables \\ Facultad de Ciencias Económicas \\ Universidad Nacional de La Plata / Buenos Aires-Argentina \\ [Recepción: Mayo de 2013/ Conformidad: Junio de 2013]
}

\section{RESUMEN}

El objetivo de este trabajo consiste en un análisis de los efectos de la actividad de las empresas de aeronavegación comercial y aquello informado o que deberían informar en sus estados financieros.

En los últimos tiempos se ha observado un gran aumento de la actividad turística. La Organización Mundial de Turismo señala que entre todas las actividades económicas, el turismo es una de las que más ha crecido en los últimos tiempos a nivel mundial y la tendencia es que así continúe. Las contribuciones que esta actividad genera son múltiples y cubren diversos ámbitos: el económico, el social, el cultural, el medio ambiental, etc. Esto lleva a que cada vez sean más los países que utilizan esta industria como apoyo de sus estrategias de desarrollo.

Cabe preguntarse, si lo antes expuesto producirá incrementos de componentes nocivos en la atmósfera produciendo un cambio climático y además, si hay agentes que están diseñando planes para evitarlo, introduciendo nuevos conceptos de motores de aviones en lo relativo a su fabricación, operación y mantenimiento. Asimismo, desde el punto de vista de la profesión contable, nos surgiría la pregunta: ¿las empresas aéreas brindan información en los estados contables sobre el impacto producido en el medioambiente originado por la actividad de vuelo?

Palabras claves: aeronavegación comercial, turismo, actividades económicas, medioambiente.

\begin{abstract}
The aim of this paper is an analysis of the effects of the activity of commercial airline companies and that informed or should report in their financial statements. In recent times there has been a large increase in tourism. The World Tourism Organization says that among all economic activities, tourism is one of the fastest growing in recent years worldwide, and the trend is to remain so. The contributions generated by this activity are numerous and cover various fields: economic, social, cultural, environmental media, etc.. This leads to more and more countries using this industry in support of their development strategies. One wonders, if the above produce harmful components increases in the atmosphere causing climate change and also, if there are agents who are developing plans to avoid introducing new aircraft engine concepts regarding manufacture, operation and maintenance. Also, from the point of view of the accounting profession, we arise the question: What the airlines provide information in the financial statements on the impact produced on the environment caused by flight activity?
\end{abstract}

Keywords: commercial aviation, tourism, economic activities, environment.

* Doctor en Ciencias de Dirección- Universidad Argentina de la Empresa. Máster en Administración de Negocios, Merrick School of Business University of Baltimore (EE.UU.). Especialista en Gestión Universitaria, Universidad Nacional de Mar del Plata. Contador Público, Universidad de Buenos Aires. E-mail: rpahlen@uolsinectis.com.ar 


\section{INTRODUCCIÓN}

Las empresas de aeronavegación han sido durante años el signo de expresión objetiva del avance tecnológico de nuestros días. Las comunicaciones internas y externas de un país requieren de flotas aéreas siempre en aumento para satisfacer demandas de pasajeros que se trasladan de un punto a otro con una optimización del tiempo disponible.

Las Cámaras de Turismo destacan entre las características especiales de la actividad económica del turismo y viajes su "transversalidad", por cuanto motiva y genera importantes demandas cruzadas con otros sectores de la economía nacional. Asimismo, le asignan una significativa importancia al análisis de su dinámica, porque amplifica los movimientos de la actividad económica general, sin dejar de lado el concepto de sostenibilidad ambiental, es decir un desempeño relativamente limpio con referencia a la contaminación y conservación de los recursos naturales.

El turismo es un fenómeno mundial que crece a pasos agigantados, para el año 2020, la OMT proyecta que viajarán por el mundo 1.6 billones de personas. Por otra parte, el constructor aeronáutico estadounidense Boeing anunció en abril del corriente año una inversión de 1.000 millones de dólares y la creación de 2.000 puestos de empleo en una planta en Carolina del Sur (sureste de Estados Unidos), donde fabrica su avión 787 .

Conociendo este significativo movimiento aéreo, cabe preguntarse, si lo antes expuesto producirá incrementos de componentes nocivos en la atmósfera produciendo un cambio climático y, además, si hay agentes que están diseñando planes para evitarlo, introduciendo nuevos conceptos de motores de aviones en lo relativo a su fabricación, operación y mantenimiento. Asimismo, desde el punto de vista de la profesión contable, nos surgiría la pregunta: ¿las empresas aéreas brindan información en los estados contables sobre el impacto producido en el medioambiente originado por la actividad de vuelo?

El objetivo de este trabajo consiste en un análisis de los efectos de la actividad de las empresas de aeronavegación comercial y aquello informado o que deberían informar en sus estados financieros.

\section{EL CAMBIO CLIMÁTICO Y LASEMPRESAS AÉREAS}

Debido a la necesidad urgente de disminuir las emisiones de gases de efecto invernadero, es necesario promover el uso de energías que no emitan los gases que provocan el cambio climático global.

El Panel Intergubernamental del Cambio Climático patrocinado por Naciones Unidas prevé que las temperaturas medias del planeta aumentarán hasta $5.8^{\circ} \mathrm{C}$ durante este siglo. Muchos países aceptan actualmente que las emisiones de gases de efecto invernadero deben recortarse de manera drástica para limitar los efectos ambientales negativos que el calentamiento global provocaría.

A partir del protocolo de Kyoto de 1997, que requiere una emisión global de gases de efecto invernadero del 5.2\% para el periodo 2008-2012 respecto de los niveles de 1990, se han introducido una serie de objetivos de reducción a escala regional y nacional. No obstante, no debemos descartar que el transporte aéreo directamente y la aeronáutica, de modo indirecto, son incluidos entre los generadores del cambio climático.

\section{LAS EMPRESAS DE AERONAVEGACIÓN Y LA INFORMACIÓN CONTABLE}

El tema ambiental es una cuestión que el sistema contable no puede ignorar y es por ello que consideramos de suma importancia en este trabajo abordar la necesidad de construir un nuevo esquema que vincule de una manera sistémica los problemas sociales actuales y el deterioro ambiental, en este caso originado en la prestación de un servicio representado por la actividad comercial de empresas aéreas, así como también la respuesta que estos entes asumen y lo que consideramos que debería ser informado en sus estados financieros.

Es sabido que en los últimos años se ha tomado conciencia de la importancia de la naturaleza para el desarrollo de la humanidad y de cómo aquellos actos denominados medios de subsistencia del ser humano contribuyen a su deterioro; sin embargo algunos estudios han demostrado que desde que el hombre habita la Tierra se ha visto un uso desmesu-

58/ QVIPURAMAYOC | Vol. 21(39) 2013 
rado e inconsciente del medio ambiente y como respuesta a esta situación, desde finales del siglo XIX, han surgido grupos de preservación del medioambiente.

Dado que la degradación del medio ambiente conlleva a una pérdida de calidad de vida de los seres humanos, se han buscado mecanismos que ayuden a su mejoramiento y recuperación, teniendo en cuenta que este es un tema que afecta a toda la sociedad, las empresas como explotadoras de recursos naturales han tenido que replantear las formas de producción y prestación de servicios, participando así en el cuidado y preservación del medio ambiente. Para ello debieron incurrir en algunos costos que deben ser captados por el sistema contable, de tal manera que surgen nuevas estructuras que buscan dar cuenta de la utilización, desgaste y pérdida de los recursos naturales, naciendo así el nuevo término "Contabilidad ambiental" y en muchos aspectos esa especialidad se ha incorporado a la rama de la "Contabilidad Patrimonial o Financiera", al reconocer el desgaste dentro de un determinado periodo, como así también activos intangibles, elementos de propiedades, planta y equipos y deudas ciertas e inciertas.

Debido al proceso de globalización que está sufriendo el mundo y la continua evolución de la estructura financiera de los países, se ha provocado un cambio en materia económica; y no por ello se debe aducir la existencia de un vacío de las normas contables profesionales particulares, pues en dichos casos será necesario recurrir al Marco Conceptual y reconocer aquellas variaciones patrimoniales que puede generar el ente con su actividad y así brindar información a terceros -usuarios tipo- que refleje fielmente la realidad económica del ente.

\section{a) Marco conceptual}

El marco conceptual para la información financiera (IASB) busca acercar a la contabilidad con la economía, es así que señala como objetivo el exponer "información sobre los recursos económicos de la entidad y los derechos de los acreedores contra la entidad que informa. Los informes financieros también proporcionan información sobre los efectos de transacciones y otros sucesos que cambian los recursos económicos y los derechos de los acreedores de una entidad que informa. Ambos tipos de información proporcionan variables útiles para tomar decisiones sobre proporcionar recursos a una entidad".

En ese mismo marco conceptual, que trata cuestiones clave como las características cualitativas de la información financiera, una de las fundamentales es la "representación fiel" (en otros países, aproximación a la realidad) donde se destaca que los estados financieros, para ser creíbles, deben presentar descripciones y medidas contables que guarden una correspondencia razonable con los fenómenos que pretenden describir.

Los usuarios tipo -Inversores- necesitan evaluar razonablemente los riesgos del ente en marcha, es decir si cumple con la capacidad de generar ingresos que ayuden a recuperar su inversión inicial.

\section{b) Hipótesis de negocio en marcha}

Los entes definen sus objetivos, y para alcanzarlos deben realizar actividades que conforman su ciclo operativo, que en virtud de su dinámica se superponen continuamente. Ese patrimonio en constante cambio continuará en el futuro, no vislumbrándose intención de disminuir la escala de sus operaciones. Ello nos lleva a pensar que el impacto positivo sobre el patrimonio de estos entes se estaría verificando en períodos distintos a aquellos en los que se devengan los resultados negativos relacionados con las consecuencias desfavorables sobre el medioambiente que esta actividad genera.

Serán los usuarios tipo, los inversores y acreedores, aquellos interesados en conocer la habilidad del ente para generar ingresos netos de caja positivos y las decisiones económicas serán tomadas a partir de la evaluación de la capacidad de la mencionada organización para generar flujos de efectivo en el futuro. $\mathrm{Al}$ respecto el marco conceptual para la Información Financiera (IASB) prescribe que "los estados financieros se preparan normalmente bajo el supuesto de que una entidad está en funcionamiento, y continua- 
rá su actividad dentro del futuro previsible. Por lo tanto, se supone que la entidad no tiene la intención ni la necesidad de liquidar o recortar de forma importante la escala de sus operaciones. Si tal intención o necesidad existiera, los estados financieros pueden tener que prepararse sobre una base diferente, en cuyo caso dicha base debería revelarse". )" (Marco Conceptual para preparación y presentación de la estados financierosIASB, Capitulo 4).

El objetivo de la gestión empresarial es la creación de valor y los estados financieros deberían proveer información a los usuarios tipo, por ejemplo a los inversores actuales y potenciales (usuarios de riesgo) sobre la probabilidad de que el ente pague dividendos, como así también otros datos necesarios para tomar decisiones de comprar, retener o vender sus participaciones.

La idea de empresa en marcha es que el ente tiene una vida indefinida, que habrá de continuar operando, utilizando sus activos, para llevar adelante el ciclo operativo, y que por el tipo de actividad que desarrolla, no tiene en venta los aviones, sino solamente la prestación de un servicio.

Entre las características cualitativas fundamentales de la información contenida en los estados financieros, a efectos de lograr que ella sea útil a los usuarios tipo, debe cumplirse con el de "relevancia": "la información financiera relevante es capaz de influir en las decisiones tomadas por los usuarios. La información puede ser capaz de influir en una decisión incluso si algunos usuarios eligen no aprovecharla o son ya conocedores de ella por otras fuentes". (Marco Conceptual para la Información financiera- IASB, Capitulo 3).

Esto en general ocurre con la información financiera que: "es capaz de influir en las decisiones si tiene valor predictivo, valor confirmatorio o ambos. $\mathrm{La}$ información financiera tiene valor predictivo si puede utilizarse como una variable en los procesos utilizados por usuarios para predecir resultados futuros. La información financiera con valor predictivo se emplea por los usuarios para llevar a cabo sus propias predicciones. La información financiera tiene valor confirmatorio si proporciona información sobre (confirma o cambia) evaluaciones anteriores." ${ }^{\prime 3}$
Comprendemos que el mundo de los negocios presenta riesgos, pero debemos destacar que el requisito anteriormente enunciado solamente se respetaría cuando el ente reconoce la variación patrimonial modificativa negativa que causaría el aumento del tráfico aéreo y su impacto en el medio ambiente, ya que la combustión genera óxido de nitrógeno, dióxido de sulfuro y dióxido de carbono que, combinados con la humedad de la atmósfera contribuyen a la formación de la lluvia ácida. Es por lo antedicho que el transporte aéreo directamente y la aeronáutica, de modo indirecto, son incluidos entre los generadores del cambio climático.

Cabe entonces preguntarnos si frente a tal situación no sería necesario, mientras no se logre mitigar el impacto con la prestación de un servicio aéreo que reduzca la emisión de gases, producto de inversiones para poner en servicio equipos de última generación, registrar un resultado negativo con contrapartida en el pasivo, luego de verificarse las condiciones necesarias para el reconocimiento de tal situación.

El término "ganancia" no resulta abarcativo del problema global, ya que las decisiones de distribución de resultados no son las únicas que afectan al ente, y por lo tanto cualquier tipo de desvío o incorrección en la determinación de la ganancia o pérdida de un ejercicio, derivará en consecuencias no deseadas a la hora de utilizar la información contable.

Cabe agregar que cuando nos referimos a información contable debemos admitir que solo intenta reflejar la realidad económica circundante y de ningún modo lo inverso, como en algunos casos se pretende demostrar. Para algunos, la culpa de todos lo males se origina en la supuestamente incorrecta información que provee la contabilidad, como si esta fuera capaz de modificar lo que sucede a su alrededor, o fuera previa a los hechos que refleja.

No olvidemos la característica cualitativa de la información -predictiva- a que hace referencia el marco conceptual, por lo tanto el sistema contable debe ser apto para elaborar un producto final donde la información sea un elemento de anticipación y diagnóstico de situaciones futuras esperadas. Cabe 
destacar que la elaboración de información proyectada es cada día más requerida por los administradores de los entes y por los terceros involucrados.

A pesar de ello, mantenemos nuestra afirmación en el sentido de que dicha información no modifica la realidad económica, sino tan solo la anticipa, dentro de los parámetros estipulados por los entes: "Sobre la base de un razonamiento un poco más complejo podría establecerse algún tipo de relación ente los hechos que se muestran en una proyección y los que luego suceden, intentando luego demostrar que estos últimos tienen influencia sobre los primeros. Como se suele decir popularmente, si repetimos un suficiente número de veces que algo va a suceder, es posible que suceda. No consideramos que ello sea adecuado dentro del esquema de análisis de la información contable. No importa cuantas veces se emitan informaciones erróneas o distanciadas de lo que va a suceder, los hechos llegarán inexorablemente y generarán una nueva información, esta vez basada en la realidad irrefutable". (Chyrikins et al, 2000).

\section{TERMINOLOGÍA CONTABLE: PROVI- SIONES, OBLIGACIONES ACUMULADAS, RESERVAS}

Los pasivos están representados por las obligaciones de entregar dinero, bienes o prestar servicios, donde la cancelación de la obligación es cierta o altamente probable. $\mathrm{Al}$ respecto, el marco conceptual para la información financiera (IASB-2010) define al pasivo como "una obligación presente de la entidad, surgida a raíz de sucesos pasados, al vencimiento de la cual, y para cancelarla, la entidad espera desprenderse de recursos que incorporan beneficios económicos".

Los compromisos ciertos están constituidos por aquellos asumidos por el ente, cualquiera fuera su origen y que ineludiblemente deben ser cumplidos, por ello lo de "ciertos" y están integrados por lo que conocemos como deudas, sean bilaterales o unilaterales, formalizadas o no. Por lo tanto incluimos las deudas y las obligaciones acumuladas o devengadas.

La denominación "obligaciones acumuladas" representa cargos ciertos de compromisos devengados no exigibles a la fecha de cierre de ejercicio, que se transformarán en deudas (pasivos exigibles) a corto plazo, pero al cierre del ejercicio hay ausencia del vinculo jurídico entre deudor y acreedor. Ejemplos de ellas son las relacionadas con impuestos, sueldo anual complementario y sus cargas sociales, entre otras.

En general los pasivos se consideran ciertos, pues los estados financieros (contables) deberían confeccionarse bajo el supuesto que el ente cancelará todos los pasivos asumidos provenientes de disposiciones legales o contractuales, pero no se debe ignorar que hay otros que permiten suponer que el ente aceptará ciertas responsabilidades futuras frente a terceros y son las denominadas obligaciones implícitas.

Atento la existencia de incertidumbre que rodea los negocios desarrollados por el ente, donde podríamos definir: "Incertidumbre: falta de certidumbre, duda, perplejidad. Certidumbre: seguro, obligación de cumplir alguna cosa" (DRAE, 2001).

Surgirán pasivos contingentes, originados entre otras causas en:

a) Garantías por defectos de producción que pueden ejecutarse o no.

b) Juicios contra el ente cuya resolución sea incierta y que la información contable brindada a terceros no debe ignorar. Por ello, sería adecuado:

a) Reconocer en el período de su nacimiento situaciones inciertas, efectuando para ello las estimaciones pertinentes, cuando ellas sean probables y de medición confiable, cuyo hecho generador ya haya ocurrido y además informarse en nota en los estados financieros.

b) Las remotas no deberían contabilizarse por medio de una variación patrimonial modificativa ni exponerse en nota.

c) Las restantes: probables no reconocidas y no probables ni remotas, deberían informarse en nota a los estados financieros.

Por lo tanto, la información complementaria debería informar sobre las contingencias no remotas al cierre del ejercicio y su tratamiento contable.

Estas condiciones serían válidas para las contingencias negativas y la doctrina propone que debe reconocerse una "provisión" cuando: 
a) el emisor de los estados financieros tiene una obligación (legal o implícita) resultante de un hecho ya ocurrido

b) es probable que para cancelarla se requiera una salida de recursos con valor económico

c) el importe de la obligación puede estimarse de manera fiable

Obviamente, similar tratamiento tendrán las contingencias ambientales (entre otras, obligaciones eventuales por las multas medioambientales o por los costos de reparación de los daños ocasionados).

Si bien, la NIC 37 exige que una provisión sea reconocida contablemente contra resultados, si el ente tiene una obligación de transferir beneficios económicos futuros y no se le presenta otra alternativa, toda vez que haya establecido una política de normas de gestión ambientales (basadas en la renovación de la flota, revisión e innovación en políticas de ahorro de combustible y concientización cultural en defensa del medio ambiente) y se haga pública su postura de atenuar los efectos negativos ocasionados, correspondería constituir también una provisión, porque existe una obligación implícita por el interés de mantener la imagen de la organización y sus relaciones con la sociedad en la que opera. En caso contrario, en nuestra opinión, se estarían omitiendo pasivos, distribuyendo resultados no genuinos y haciendo peligrar el concepto de empresa en marcha.

En relación con el significado de "reserva", el Diccionario de la Real Academia Española indica: "Guarda o custodia que se hace de algo, o prevención de ello para que sirva a su tiempo.” Asimismo del verbo reservar, podríamos tener en cuenta las siguientes acepciones:

- Guardar algo para lo futuro

- Dilatar para otro tiempo lo que se podía o se debía ejecutar o comunicar al presente

- Destinar un lugar o una cosa, de un modo exclusivo, para uso o persona determinados."

Desde el punto de vista contable, influenciado por el concepto jurídico de capital, constituyen reservas los elementos del patrimonio social que no corresponden al capital (aporte de los propietarios) y que constituyen resultados que no se distribuyen entre los socios, formando parte de la autofinanciación por resultados no distribuidos o financiación propia de la empresa. Por lo tanto, las reservas representan resultados con algún tipo de restricción a su distribución, originada en disposiciones legales, en el estatuto o por voluntad de la Asamblea de Accionistas. Debemos tener en cuenta que si su origen se encuentra en resultados positivos, solamente se las constituye y se restringe la distribución de ganancias cuando ellas surjan de la determinación efectuada por el ente al finalizar el ejercicio económico.

Cabe agregar lo siguiente: "los destinatarios naturales de los beneficios de una sociedad son sus socios o accionistas; a pesar de ello, diversos motivos pueden llevar a los administradores a no distribuir los beneficios en su totalidad. Pueden existir, por ejemplo, razones vinculadas con el futuro societario, el que no siempre está asegurado contra las contingencias del negocio, así como la intención de encarar nuevas inversiones o expandir las actividades, etcétera. En consecuencia, la asamblea de accionistas puede decidir la separación de ciertos importes de las ganancias no asignadas y afectarlos a un destino específico, es decir, restringir su posible distribución. Esto se conoce con la denominación de constitución de reservas y puede derivar de la ley, del estatuto o contrato social o emanar, simplemente, de la libre voluntad de la asamblea." (Pahlen et al., 2009)

\section{CONCLUSIONES}

El tema ambiental es una cuestión que el sistema contable no puede ignorar y es por ello que consideramos de suma importancia en este trabajo abordar la necesidad de construir un nuevo esquema que vincule de una manera sistémica los problemas sociales actuales y el deterioro ambiental, en este caso originado en la prestación de un servicio representado por la actividad de empresas aéreas, como así también la respuesta que estos entes asumen y lo que consideramos que debería ser informado en sus estados financieros.

Los entes definen sus objetivos, y para alcanzarlos deben realizar actividades que conforman 
su ciclo operativo, y en virtud de su dinámica, esos ciclos se superponen continuamente. Ese patrimonio en constante cambio continuará en el futuro, no vislumbrándose intención de disminuir la escala de sus operaciones. Ello nos lleva a pensar que el impacto positivo sobre el patrimonio de estos entes se estaría verificando en períodos distintos a aquellos en los que se devengan los resultados negativos relacionados con las consecuencias desfavorables sobre el medioambiente que esta actividad genera.

Asimismo, hemos detallado las diferencias terminológicas entre obligaciones acumuladas, provisiones y reservas. Frente a los niveles de emisiones vertidos a la atmósfera y la aplicación de nuevas tecnologías podríamos inferir -en primer lugar- la necesidad de constituir una reserva para la renovación de gran parte de los activos fijos (material de vuelo), y cuando ello suceda, desafectarla. Sin embargo, esa reserva solamente se constituiría si el ente generará ganancias, por ser los únicos resultados que se podrían restringir en su distribución, en caso de ser aprobada tal restricción por la Asamblea de Accionistas.

Cabe destacar, en relación con el reconocimiento contable de hechos inciertos desfavorables, que el Marco Conceptual de la Información Financiera IASB- indica que "ciertos pasivos solo pueden medirse utilizando un alto grado de estimación".

No debemos ignorar que la incertidumbre y el riesgo que tiene el negocio de la aeronavegación comercial pueden derivar en probables efectos negativos sobre el medioambiente. La probabilidad de que dichos efectos se concreten es necesariamente alta y la cuantificación en moneda de cuenta debería ser efectuada de una manera objetiva, representada, por ejemplo, por las obligaciones eventuales emergentes de sanciones medioambientales o por los costos de adecuación a las normativas de protección ambiental.

Si bien, las normas contables exigen que una provisión sea efectuada solamente si el ente tiene una obligación de transferir beneficios económicos futuros y no se le presenta otra alternativa, toda vez que haya establecido una política de normas de gestión ambientales, (basada en la renovación de la flota, revisión e innovación en políticas de ahorro de combustible y concientización cultural en defensa del medio ambiente) y se haga pública su postura de atenuar los efectos negativos ocasionados, correspondería constituir una provisión, porque existe una obligación implícita por el interés de mantener la imagen de la organización y sus relaciones con la sociedad en la opera.

En nuestra opinión, de no proceder de acuerdo con lo antes expuesto, se estarían omitiendo pasivos, distribuyendo resultados no genuinos y haciendo peligrar el concepto de hipótesis de empresa en marcha.

\section{BIBLIOGRAFÍA}

1. Diccionario de la Lengua Española. (2001). Real Academia Española. Madrid. Vigésima segunda edición.

2. Pahlen Acuña, Ricardo y Fronti de García, Luisa, (2004). Contabilidad Social y Ambiental. Editorial Macchi. Buenos Aires. Argentina.

3. Pahlen Acuña, Ricardo y Fronti de García, (2005). Contabilidad ambiental. Un nuevo segmento. Ediciones cooperativas. Buenos Aires.

4. Pahlen Acuña, Ricardo y Fronti de García, Luisa (2007). Contabilidad ambiental. Segmento para el siglo XXI. Ediciones cooperativas. Buenos Aires.

5. Pahlen, Ricardo J.M, Campo Ana M, Chávez Osvaldo A, Fronti Luisa, Helouani Ruben y Viegas Juan C. Contabilidad. Pasado, Presente y Futuro. La Ley -2009

6. Fowler Newton, Enrique. Normas Internacionales de Contabilidad Financiera. La Ley. 2006

7. Chyrikins, Héctor y otros. Los resultados en el contexto de modelos contables alternativos. Trabajo presentado a las XXI Jornadas Universitarias de Contabilidad. UADE. 2000

8. Marco Conceptual de la Información Financiera- IASB - 2010

9. Normas Internacionales de Información Contable- IASB NIC 36, 37 y 38 
10. http://www.coiae.com/presentacion/Info Avion_Verde.asp

11. http://www.airportnewsezeiza.com/paginas/ notas/mercado.html

12. http://www.pactoglobal.org.ar/userfiles/file/ Perfil_de_las_empresas_adheridas.pdf
13. http://www.unglobalcompact.org/languages/ spanish/Implementando_el_Pacto_Mundial.pdf

14. http://www.camaraargentinadeturismo.travel/ CAT/INSTITUCIONAL/publicaciones.asp 